\title{
Clinical Characteristics, Management, and Length of Hospital Stay Between Patients with New-Onset and Acute Decompensated Chronic Heart Failure: A Prospective Cohort Study in Ethiopia
}

\author{
Bekalu Kebede' \\ Bekalu Dessie (D) \\ Melese Getachew ${ }^{2}$ \\ Yalew Molla (iD) ${ }^{3}$ \\ Bereket Bahiru (D) ${ }^{4}$ \\ Haile Amha ${ }^{5}$ \\ 'Clinical Pharmacy Unit, Pharmacy \\ Department, Health Science College, \\ Debre Markos University, Debre Markos, \\ Ethiopia; ${ }^{2}$ Pharmaceutics Unit, Pharmacy \\ Department, Health Science College, \\ Debre Markos University, Debre Markos, \\ Ethiopia; ${ }^{3}$ Pharmacology Unit, Pharmacy \\ Department, Health Science College, \\ Debre Markos University, Debre Markos, \\ Ethiopia; ${ }^{4}$ Social Pharmacy Unit, \\ Pharmacy Department, Health Science \\ College, Debre Markos University, Debre \\ Markos, Ethiopia; ${ }^{5}$ Department of \\ Nursing, College of Health Science, \\ Debre Markos University, Debre Markos, \\ Ethiopia
}

Background: Because heart failure with de novo and acute decompensated chronic heart failure are different clinical entities, clinical characteristics, management, and length of hospital stay may also vary accordingly.

Objective: To compare clinical characteristics, management, and length of hospital stay among adult heart failure patients with new-onset and acute decompensated chronic heart.

Method and Participants: Prospective cohort study was conducted from 01 November 2019 to 30 April 2021 at Debre Markos Comprehensive Specialized Hospital. A total of 228 heart failure patients who fulfill inclusion criteria were included in this study. A structured data collection tool was used to collect all necessary data. Data were analyzed using Statistical Package for Social Science (SPSS) version 21.0. Bivariate and multivariate logistic regression analyses were used and P-value, $<0.05$ was considered statistically significant.

Results: Among the 228 participants, $126(55.3 \%)$ were females with a mean age of $53.31 \pm$ 15.68 years. Of the study participants, $131(57.5 \%)$ were presented with acute decompensated chronic heart failure. The median length of hospital stay was 12 days (interquartile range, 8-18). De novo heart failure patients spent less time in hospital [11 days (interquartile range, 6-16) vs 13 days (interquartile range, 9-20) in acute decompensated chronic heart failure, $\mathrm{P}=0.004$ ]. Mean systolic blood pressure $(\mathrm{P}=0.006)$, acute decompensated chronic heart failure $(\mathrm{P}<0.000)$, diabetes mellitus comorbidity $(\mathrm{P}=0.025)$, and the use of angiotensin receptor blockers $(\mathrm{P}=$ 0.042 ) were independent predictors of prolonged hospital stay. During hospitalization, digoxin $(61.1 \%)$ was the most frequently prescribed in de novo heart failure while diuretics $(63.3 \%)$ were the most common in acute decompensated chronic heart failure at discharge.

Conclusion: Heart failure patients were presented with diverse clinical characteristics. Length of hospital stay was higher in patients with acute decompensated chronic heart failure. Initiation of treatment must take into account the heterogeneity of each patient.

Keywords: heart failure, characteristics, management, hospital stay

\section{Introduction}

Heart failure (HF) is a great public health concern, because of its increasing prevalence and associated high morbidity, mortality, and health-related costs. ${ }^{1}$ Currently, it is one of the major clinical challenges in developing countries due to the aging of the population, better control of communicable diseases, and malnutrition. ${ }^{1,2}$ In sub-Saharan Africa, HF affects young and middle-aged adults
Department of Pharmacy, College Health Sciences, Debre Markos

University, P.O. Box 269, Debre Markos, Ethiopia

Tel +251921286140

Email bekalukebede19@gmail.com 
$($ mean $=53$ years $)$ most likely presented with New York Health Association functional class (NYHA) IV as compared with those from Asia, the Middle East, and South America. ${ }^{3}$ In Ethiopia, as in many developing countries, there is a burden of epidemiological transition from communicable to non-communicable diseases (NCD) in the past decade. ${ }^{4}$ Moreover, in developing countries priority is given to acute disorders, child and maternal health care, and control of communicable diseases, giving very little emphasis on chronic NCD. ${ }^{3,4} \mathrm{HF}$ is the second most diagnosed cardiovascular disorder (CVD) in Ethiopia following hypertension $62.3 \%$ and $23.9 \%$, respectively. ${ }^{5}$

$\mathrm{HF}$ is one of the primary reasons for regular hospital visits and re-admissions, accounting for about 3 to $7 \%$ of admissions in Africa. ${ }^{1,4}$ The in-hospital mortality rate of HF ranges from $9 \%$ to $12.5 \%$, which is considerably higher than in developed countries $(3.8-6.4 \%) .{ }^{3}$ During hospitalization, HF patients with new-onset and acute decompensated chronic heart failure (ADCHF) are presented with different clinical profiles. ${ }^{6,7}$ Management also varies based on the heterogeneity of $\mathrm{HF}^{8,9}$ Furosemide, beta-blockers, angiotensin-converting enzyme inhibitors (ACEIs), spironolactone, digoxin, angiotensin receptor blockers (ARBs), nitrates, and hydralazine are most commonly prescribed medication for heart failure patients. $^{11-14}$

Even though HF data from the western world are available, contemporary data from low- and middle-income countries including Ethiopia are sparse regarding presentation, management, and outcomes of patients hospitalized with HF. ${ }^{7,10}$ In addition, the risk profile of HF, practice patterns, and health-care resources in developing countries are different from those of western countries. ${ }^{15-17}$ Therefore, it is important to develop appropriate predictive models that might help to stratify HF patients, improve their management and follow-up strategies in high-risk patients to improve their outcome and decrease expenditure associated with HF. As a result, this prospective cohort study was conducted to assess the clinical characteristics, management, and outcomes among patients with de novo and ADCHF.

\section{Methods}

\section{Study Period and Area}

This study was conducted among hospitalized heart failure patients at Debre Markos Comprehensive Specialized
Hospital (DMCSH) from November 01, 2019, to April 30, 2021.

\section{Study Design}

A prospective cohort study was employed.

\section{Study Population}

Patients with a documented diagnosis of heart failure with age $\geq 18$ years, and who had complete medical records were included in the study. We excluded HF patients who transferred to other hospitals for further treatment, and patients with confirmed concomitant malignancy or World Health Organization stage 4 human immune deficiency (HIV) infections. Because this group of patients had already extended the duration of hospitalization, it was difficult to assess similarly to that of other participants. All adult patients admitted to the hospital with a diagnosis of heart failure during the study period and fulfilled inclusion criteria were recruited.

\section{Data Collection Instrument and}

\section{Procedures}

A structured data collection tool, which includes a questionnaire and data abstraction format, was used to extract all necessary information. Data collections and followups were performed from November 01, 2019, to 30 April 2021. Data were collected from parents by face-toface interviews. Secondary data such as prescribed drugs, laboratory and diagnostic results, and co-morbid illnesses were collected from patients' medical charts daily. HF was defined, classified and its management was evaluated according to the American College of Cardiology/American Heart Association (ACC/AHA) guideline 2017, and events were classified as new-onset with no prior history of HF (de novo HF) and ADCHF (worsening HF). ${ }^{12}$ The ejection fraction of the participants was measured using an echocardiogram during their first admission and categorized to either reduced ejection fraction; if $\mathrm{EF}<\mathrm{b} 50 \%$ or preserved $\mathrm{EF}$; if $\mathrm{EF} \geq 50 \%{ }^{14}$

\section{Data Quality Assurance}

Data collectors were trained by the principal investigator about the data collection method, the appropriate use of the data collection instruments, and the confidentiality of the collected data. The data collectors were recruited based on their experience in medical ward practice that is capable of assessing patients' past medication experience. The questionnaire was translated to the local language, Amharic, for 
consistency. A pre-test of data collection format was performed on $5 \%$ of the sample before conducting the study. Then, the final tool was developed with some modifications after reviewing the results of the pre-test. The quality of data was also checked before data entry.

\section{Data Processing and Analysis}

The data were entered into Epidata version 3.1 and exported to the Statistical Package for Social Science (SPSS) version 21.0 for analysis. Continuous variables were presented as means (standard deviation) and interquartile range. Categorical variables were presented as frequency and percentages. Categorical variables were compared by using the chi-square test and continuous variables by the $t$-test. Bivariate logistic regression analysis was done to see associations between the dependent and independent variables. Then, independent variables having a P-value of less than 0.25 were included in multivariate logistic regression analysis to identify independent predictors of prolonged hospital stay. Those variables having $\mathrm{P}$-value, $<0.05$ were considered statistically significant.

\section{Operational Definitions}

Length of hospital stay: Is the difference in days between discharge and admission dates of patients. ${ }^{18}$
The short length of stay: Defined as hospital stay less than media of hospital stay ( $<12$ days) otherwise defined as prolonged hospital stay. ${ }^{12}$

Co-morbid: It is the presence of one or more additional disorders (diseases) co-occurring with a primary disease or disorder. $^{15}$

Physically active: Classified if they reported that they exercise more than 30 minutes, more than 5 days/week. ${ }^{19}$

Preserved ejection fraction: Was defined as left ventricular ejection fraction $\geq 50 \%$ measured using an echocardiogram on the first day of admission. ${ }^{20}$

Hypertension: Defined when blood pressure $\geq 130 / 80$ $\mathrm{mmHg}$ for all age groups and comorbidities. ${ }^{14}$

\section{Result}

\section{Demographic Characteristics of Study Participants}

A total of 251 patients were admitted to DMCSH hospital with the diagnosis of HF during the study period. Two hundred forty-six HF patients were fulfilled the inclusion criteria and invited to participate. Finally, 228 participants were included in the final analyses (Figure 1). Of these, $126(55.3 \%)$ were females. The mean age of participants was $53.31 \pm 15.68$ and patients with de novo HF were younger as compared to patients with ADCHF. More than one-half $(62.5 \%)$ of ADCHF participants were from rural

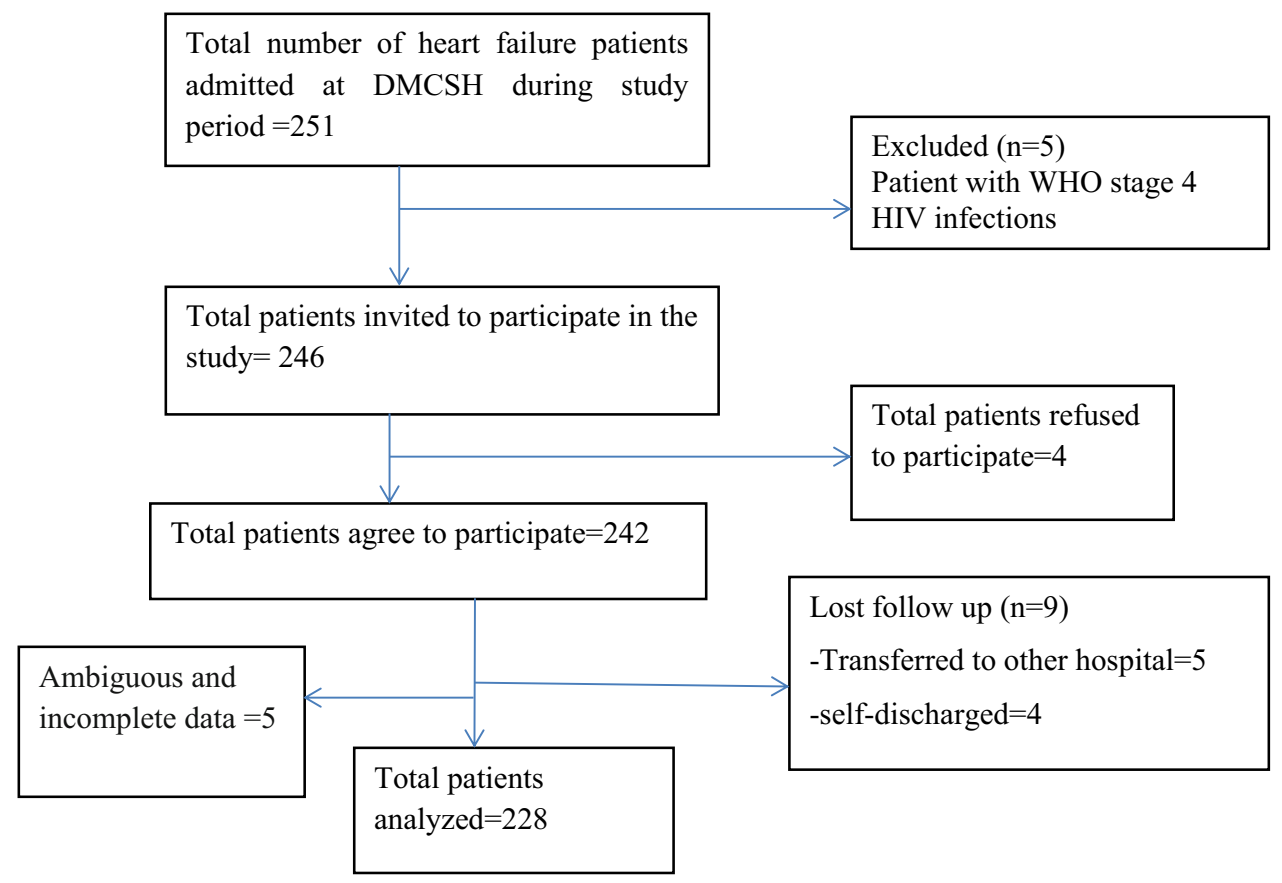

Figure I Heart patient selection flow chart at Debre Markos Comprehensive specialized Hospital medical ward from November 0I, 2019 , to April 30, 202I. 
centers, and $62.3 \%$ of this group were non-government employers. About half of patients with de novo AHF were physically active but they are more coffee drinkers than patients with ADCHF (Table 1).

Out of the total participants 131 (57.5\%), were presented with ADCHF and $42.5 \%$ of patients had new-onset HF. Almost all HF patients were hospitalized with advanced stage of heart failure (93\% of NYHA class IV and $96.5 \%$ of ACC/AHA stage C respectively). In physical examination, an abnormal S3 and S4 sound (gallop) $(56.2 \%)$ and reduced ejection fraction $(53.7 \%)$ were more frequent in de novo HF. Similarly, mean heart rate, respiratory rate, and temperature were high among de novo $\mathrm{HF}$ as compared to ADCHF. However, raised JVP (71.1\%), pulmonary congestion $(70.1 \%)$, and fatigability (62.1\%) were more frequently experienced in ADCHF. Ischemic heart disease and atrial fibrillation were the most common co-morbid conditions in de novo HF. However, asthma/COPD, dyslipidemia, and prior history of stroke were most frequent in ADCHF (Table 2).

Medication use pattern showed that digoxin (61.1\%) and ARBs (53.8\%) were the most frequently prescribed class of medication during hospitalization of de novo HF patients. On the other hand, the use of diuretics (57.1\%) and antiplatelet (66.7\%) were higher among ADCHF than de novo HF. In the same ways, the use of diuretics and beta-blockers was significantly higher in ADCHF than de novo HF $(\mathrm{p}<0.005)$ at discharge (Table 3$)$.

\section{Length of Hospital Stay and Its Determinants Among Heart Failure Patients}

The median time spent in the hospital was 12 days (IQR 8-18). There were 131 (58.3\%) participants with LOS with $\geq$ a median hospital stay (12 days). Of these, 47 (35.3\%) were de novo HF patients and 86 (64.7\%) were ADCHF. De novo HF patients spent less time in hospital [11 (IQR 6-16) vs 13 (IQR 9-20) days, P = 0.004]. After adjusting cofounding variables, independent predictors of prolonged hospital stay are reported in Table 4. Accordingly, increasing systolic blood pressure was significantly associated with longer LOS as compared to patients within normal systolic blood pressure (AOR $1.26, \mathrm{CI}=0.99-1.53, \mathrm{P}=0.006$ ).

On the other hand, lower serum creatinine concentration was significantly associated with shorter LOS as compared to patients with elevated serum creatinine
(AOR 0.46, CI $=0.27-0.87, \mathrm{P}=0.004$ ). Participants hospitalized with $\mathrm{ADCHF}$ were 2.66 times $(\mathrm{AOR}=2.66$, $\mathrm{CI}=1.35-5.22, \mathrm{P}=0.000$ ) more likely to had prolonged hospitalization as compared to de novo HF. Similarly, the use of ARBs has increased the risk of long-term hospitalization by 2.32 times $(\mathrm{AOR}=2.32, \mathrm{CI}=1.03-5.20, \mathrm{P}=$ 0.042). HF patients who had diabetes mellitus comorbidity were 2.07 times $(\mathrm{AOR}=2.07, \mathrm{CI}=0.99-4.33, \mathrm{P}=0.025)$ more likely to had prolonged hospitalization as compared to patients without diabetes mellitus comorbidity (Table 4).

\section{Discussion}

Our finding showed that more than half of acute presentations of heart failure were decompensated, predominantly females, and almost all presented to the hospital after the symptomatic stage (with NYHA class IV and stage C) of heart failure. Patients with de novo heart failure were younger and physically active, but more likely to drink coffee. On the other hand, raised Jugular Venous Distention (JVP), pulmonary congestion, and fatigability were more frequently experienced in ADCHF. These findings were in line with other similar studies. ${ }^{13,21}$ In contrast, more than half of patients with de novo heart failure had reduced ejection fraction but had high mean heart rate, respiratory rate, and temperature as compared to ADCHF. The increase of heart rate in new-onset HF might be because of an immediate compensatory mechanism for decreasing cardiac output and the high prevalence of infection in this group. ${ }^{12,22} \mathrm{~A}$ high prevalence of comorbidities was observed in patients, particularly in the ADCHF groups. These patients were at higher risk for further deterioration and death, thus suggesting the need for implementing therapeutic strategies to improve good treatment outcomes. ${ }^{14,17}$ In this study, there was a high prevalence of asthma/COPD in ADCHF than in those with de novo HF, which was similar to reports in different studies. ${ }^{11,15}$ This might be because HF is strongly correlated with hypoxemia and airflow obstruction associated with asthma/COPD that alters structure (eg, hypertrophy or dilatation) and impaired right ventricular function secondary to pulmonary hypertension. ${ }^{11,23}$ In addition, the presence of dyslipidemia and prior history of stroke were more frequent in ADCHF. This might be due to common risk factors including dyslipidemia for heart failure and stroke. ${ }^{24}$

In this study, diuretics were the most prescribed class of medications for $\mathrm{HF}$ patients and it was higher in ADCHF groups. The use of diuretics is encouraged to get the relief of pulmonary and peripheral congestion 
Table I Demographic Characteristics of Patients Admitted with Heart Failure with De novo and ADCHF

\begin{tabular}{|c|c|c|c|c|}
\hline \multirow[t]{2}{*}{ Variables } & \multirow[t]{2}{*}{ Total $(n=228)$} & \multicolumn{3}{|l|}{ HF Category } \\
\hline & & De novo $\mathrm{HF}(\mathrm{n}=97)$ & $\operatorname{ADCHF}(n=|3|)$ & P-value \\
\hline Age(years) mean $\pm S D$ & $53.31 \pm 15.68$ & $51.84 \pm 14.94$ & $54.31 \pm 16.60$ & 0.222 \\
\hline $\begin{array}{l}\text { Sex } \\
\qquad \text { Male n (\%) } \\
\text { Female n (\%) }\end{array}$ & $\begin{array}{l}102(44.7) \\
126(55.3)\end{array}$ & $\begin{array}{l}49(48.0) \\
48(38.1)\end{array}$ & $\begin{array}{l}53(52.0 \%) \\
78(61.9)\end{array}$ & 0.131 \\
\hline $\begin{array}{l}\text { BMI }(\mathrm{kg} / \mathrm{m} 2) \\
\quad<24.9 \mathrm{n}(\%) \\
25-30 \mathrm{n} \mathrm{( \% )} \\
>30 \mathrm{n}(\%)\end{array}$ & $\begin{array}{l}147(64.5) \\
55(24.1) \\
26(11.4)\end{array}$ & $\begin{array}{l}55(37.4) \\
28(50.9) \\
14(53.8)\end{array}$ & $\begin{array}{l}92(62.6) \\
27(49.1) \\
12(46.2)\end{array}$ & 0.105 \\
\hline $\begin{array}{l}\text { Residency } \\
\text { Urban n (\%) } \\
\text { Rural n (\%) }\end{array}$ & $\begin{array}{l}108(47.4) \\
120(52.6)\end{array}$ & $\begin{array}{l}53(49.1) \\
44(37.5)\end{array}$ & $\begin{array}{l}55(50.9) \\
76(62.5)\end{array}$ & 0.051 \\
\hline $\begin{array}{l}\text { Education level } \\
\text { No formal education n (\%) } \\
\text { Primary education n (\%) } \\
\text { Secondary education n (\%) } \\
\text { Tertiary education n (\%) }\end{array}$ & $\begin{array}{l}101(44.3) \\
30(13.2) \\
40(17.5) \\
57(25.0)\end{array}$ & $\begin{array}{l}38(37.6) \\
10(33.3) \\
19(47.5) \\
30(52.6)\end{array}$ & $\begin{array}{l}63(62.4) \\
20(66.7) \\
21(52.5) \\
27(47.4)\end{array}$ & 0.186 \\
\hline $\begin{array}{l}\text { Current occupation } \\
\text { Governmental employer n (\%) } \\
\text { Non-government employer n (\%) }\end{array}$ & $\begin{array}{l}69(30.3) \\
159(69.7)\end{array}$ & $\begin{array}{l}37(53.6) \\
60(37.7)\end{array}$ & $\begin{array}{l}32(46.4) \\
99(62.3)\end{array}$ & 0.026 \\
\hline $\begin{array}{l}\text { Excessive alcohol use } \\
\text { Yes n (\%) } \\
\text { No n (\%) }\end{array}$ & $\begin{array}{l}67(29.4) \\
161(70.6)\end{array}$ & $\begin{array}{l}28(41.8) \\
69(42.9)\end{array}$ & $\begin{array}{l}39(58.2) \\
92(52.1)\end{array}$ & 0.882 \\
\hline $\begin{array}{l}\text { Salt restriction } \\
\qquad \begin{array}{l}\text { Yes } \mathrm{n}(\%) \\
\text { No } \mathrm{n}(\%)\end{array}\end{array}$ & $\begin{array}{l}127(55.7) \\
101(44.3)\end{array}$ & $\begin{array}{l}50(39.4) \\
47(46.5)\end{array}$ & $\begin{array}{l}77(60.6) \\
54(53.5)\end{array}$ & 0.277 \\
\hline $\begin{array}{l}\text { Chewing chat } \mathrm{n}(\%) \\
\text { Never } \\
\text { Sometimes } \\
\text { Always }\end{array}$ & $\begin{array}{l}182(79.8) \\
32(14.0) \\
14(6.1)\end{array}$ & $\begin{array}{l}78(42.9) \\
15(46.9) \\
4(28.6)\end{array}$ & $\begin{array}{l}104(57.1) \\
17(53.1) \\
10(71.4)\end{array}$ & 0.504 \\
\hline $\begin{array}{l}\text { Smoker n (\%) } \\
\text { Never } \\
\text { Ex-smoker } \\
\text { Current smoker n (\%) }\end{array}$ & $\begin{array}{l}179(78.5) \\
36(15.8) \\
13(5.7)\end{array}$ & $\begin{array}{l}75(41.9) \\
16(44.4) \\
6(46.2)\end{array}$ & $\begin{array}{l}104(58.1) \\
20(55.6) \\
7(53.8)\end{array}$ & 0.926 \\
\hline $\begin{array}{l}\text { Physical activity } \mathrm{n}(\%) \\
\text { Active } \\
\text { Inactive }\end{array}$ & $\begin{array}{l}79(34.6) \\
149(65.4)\end{array}$ & $\begin{array}{l}4 I(5 I .9) \\
56(37.6)\end{array}$ & $\begin{array}{l}38(48.1) \\
93(62.4)\end{array}$ & 0.037 \\
\hline $\begin{array}{l}\text { Drink coffee } \mathrm{n}(\%) \\
\text { Yes } \\
\text { No }\end{array}$ & $\begin{array}{l}\text { I38(60.5) } \\
90(39.5)\end{array}$ & $\begin{array}{l}87(63.0) \\
46(51.1)\end{array}$ & $\begin{array}{l}5 I(37.0) \\
44(48.9)\end{array}$ & 0.035 \\
\hline $\begin{array}{l}\text { Use traditional medicine } \mathrm{n}(\%) \\
\text { Yes } \\
\text { No }\end{array}$ & $\begin{array}{l}52(22.8) \\
176(77.2)\end{array}$ & $\begin{array}{l}19(36.5) \\
78(44.3)\end{array}$ & $\begin{array}{l}33(63.5) \\
98(55.7)\end{array}$ & 0.319 \\
\hline
\end{tabular}

Abbreviations: HF, heart failure; ADCHF, acute decompensated chronic heart failure; BMI, body mass index; SD, standard deviation. 
Table 2 Clinical Characteristics of Patients Admitted with Heart Failure at DMCSH

\begin{tabular}{|c|c|c|c|c|}
\hline \multirow[t]{2}{*}{ Sign and Symptoms n (\%) } & \multirow[t]{2}{*}{ Total $(n=228)$} & \multicolumn{3}{|l|}{ HF Category } \\
\hline & & De Novo HF & ADCHF & P-value \\
\hline Pulmonary congestion n (\%) & 67(29.4) & $20(29.9)$ & $47(70.1)$ & 0.051 \\
\hline Peripheral congestion n (\%) & $127(55.7)$ & $55(43.3)$ & $72(56.7)$ & 0.794 \\
\hline Cough n (\%) & $150(65.8)$ & $63(42.0)$ & $87(58.0)$ & 0.818 \\
\hline Fatigue n (\%) & $145(63.6)$ & $55(37.9)$ & $90(62.1)$ & 0.004 \\
\hline Dyspnea & $152(66.7)$ & $6 \mathrm{I}(40.1)$ & $91(59.9)$ & 0.297 \\
\hline Orthopnea n (\%) & $134(58.8)$ & $57(42.5)$ & $77(57.5)$ & 0.998 \\
\hline Raised JVP n (\%) & $45(19.7)$ & 13(28.9) & $32(7 \mid .1)$ & 0.039 \\
\hline PND n(\%) & $80(35.1)$ & $35(43.8)$ & $45(56.2)$ & 0.787 \\
\hline Gallop & $32(14)$ & $18(56.2)$ & 14(43.8) & 0.091 \\
\hline NYHA Class IV n (\%) & $212(93.0)$ & $91(42.9)$ & $|2|(57.1)$ & 0.672 \\
\hline AHA/ACC stage C n (\%) & $220(96.5)$ & $94(42.7)$ & $126(57.3)$ & 0.880 \\
\hline \multicolumn{5}{|l|}{ Ejection fraction } \\
\hline$<50 \%$ & $95(4 I .7)$ & $5 \mathrm{I}(53.7)$ & $44(46.3)$ & 0.004 \\
\hline$\geq 50$ & $133(58.3)$ & $46(34.6)$ & $87(65.4)$ & \\
\hline \multicolumn{5}{|l|}{ Vital signs } \\
\hline Heart rate (b.p.m) mean $\pm S D$ & $104.54 \pm 16.68$ & $105.51 \pm 19.64$ & $103.82 \pm 18.99$ & 0.503 \\
\hline Respiratory rate (p.m) mean $\pm S D$ & $25.4 I \pm 5.07$ & $25.44 \pm 5.42$ & $25.39 \pm 4.82$ & 0.937 \\
\hline Temperature $\left(\mathrm{C}^{0}\right)$ mean $\pm S D$ & $37.01 \pm 0.78$ & $37.15 \pm 0.76$ & $36.92 \pm 0.78$ & 0.026 \\
\hline $\mathrm{SBP}(\mathrm{mmHg})$ mean $\pm \mathrm{SD}$ & $108.9 \pm 24.00$ & $106.66 \pm 23.74$ & $109.08 \pm 24.29$ & 0.895 \\
\hline $\mathrm{DBP}(\mathrm{mmHg})$ mean $\pm \mathrm{SD}$ & $67.60 \pm 13.55$ & $67.18 \pm 13.92$ & $67.9| \pm| 3.3 \mid$ & 0.687 \\
\hline \multicolumn{5}{|l|}{ Laboratory findings } \\
\hline Serum creatinine mean $\pm S D$ & $1.34 \pm .73$ & $1.33 \pm 0.72$ & $1.34 \pm 0.72$ & 0.943 \\
\hline Blood urea nitrogen mean \pm SD & $19.02 \pm 6.6$ & $18.57 \pm 6.46$ & $|9.35 \pm 6.7|$ & 0.380 \\
\hline Hemoglobin $(\mathrm{g} / \mathrm{dl})$ mean \pm SD & $13.99 \pm 3.01$ & $13.79 \pm 2.95$ & $14.15 \pm 3.05$ & 0.158 \\
\hline RBG/FBG(mg/dl) mean $\pm S D$ & $|59.94 \pm 73.9|$ & $162.28 \pm 75.52$ & $|58.2| \pm 72.94$ & 0.682 \\
\hline Sodium level $(\mathrm{mEq} / \mathrm{L}$ mean $\pm \mathrm{SD}$ & $134.97 \pm 10.12$ & $135.92 \pm 9.55$ & $134.26 \pm 10.50$ & 0.220 \\
\hline Potassium level, $(\mathrm{mEq} / \mathrm{L})$ mean $\pm \mathrm{SD}$ & $3.58 \pm 0.91$ & $3.50 \pm 0.83$ & $3.63 \pm 0.97$ & 0.229 \\
\hline \multicolumn{5}{|l|}{ Co-morbidities } \\
\hline Ischemic heart disease $n(\%)$ & $21(9.2)$ & $12(57.1)$ & $9(42.9)$ & 0.171 \\
\hline Hypertension n(\%) & $84(36.8)$ & $3 I(37.3)$ & $52(62.7)$ & 0.266 \\
\hline Arterial fibrillation n(\%) & $50(21.9)$ & $26(74.3)$ & $9(25.7)$ & 0.040 \\
\hline Dyslipidemia n (\%) & $87(38.2)$ & $27(31.0)$ & $60(69.0)$ & 0.006 \\
\hline Diabetes mellitus n(\%) & $77(33.7)$ & $3 I(40.3)$ & $46(59.7)$ & 0.672 \\
\hline Infection n(\%) & $91(39.9)$ & $50(54.9)$ & $4 I(45.1)$ & 0.133 \\
\hline Asthma/COPD n(\%) & $63(42.5)$ & $26(4 I .3)$ & $37(58.7)$ & 0.004 \\
\hline Anemia $n(\%)$ & $63(27.6)$ & $29(46.0)$ & $34(54.0)$ & 0.409 \\
\hline Chronic renal failure $n(\%)$ & $32(14)$ & 15(46.9) & $17(53.1)$ & 0.700 \\
\hline Prior stroke $\mathrm{n}(\%)$ & $30(13.2)$ & $6(20)$ & $24(80)$ & 0.009 \\
\hline Others* & $2 \mathrm{I}(9.2)$ & $8(38.1)$ & 13(61.9) & 0.818 \\
\hline Length of hospital stay (median, IQR) & $12(8-18)$ & $\mathrm{II}(6-16)$ & $13(9-20)$ & 0.004 \\
\hline
\end{tabular}

Note: *Chronic liver disease, Thyroid disease, Deep venous thromboembolism.

Abbreviations: HF, heart failure; ADCHF, acute decompensated chronic heart failure; JVP, Jugular Venous Distention; SD, standard deviation; PND, Paroxysmal Nocturnal Dyspnea; NYHA, New York Health Association; AHA/ACC, American College of Cardiology/American Heart Association; b.p.m, beats per minutes; SBP, Systolic Blood Pressure; DBP, diastolic blood pressure; RBG/FBG, Random/fast blood glucose; COPD, chronic obstructive pulmonary disease; IQR, interquartile range.

associated with acute HF and to stabilize the patients. ${ }^{10,20}$ In contrast, during admission, the use of digoxin and ARBs were higher in the de novo group. Studies done in
Spain and Italy showed that digoxin and ARBs were most frequently prescribed among ADCHF groups. ${ }^{12,14}$ This discrepancy might be because of the higher extent of 
Table 3 Admission and Discharged Medication for Hospitalized Heart Failure Patients

\begin{tabular}{|c|c|c|c|c|}
\hline \multirow[t]{2}{*}{ Medication During Hospitalization } & \multirow[t]{2}{*}{ Overall $(\mathbf{N}=\mathbf{2 2 8})$} & \multicolumn{3}{|l|}{ HF Category } \\
\hline & & De novo AHF & ADCHF & p.value \\
\hline ACE inhibitors $n(\%)$ & $86(37.7)$ & $36(41.9)$ & $50(58.1)$ & 0.891 \\
\hline ARBs n (\%) & $65(28.5)$ & $35(53.8 \%)$ & $30(46.2)$ & 0.032 \\
\hline Beta-blockers n (\%) & $6 I(26.8)$ & $26(42.6)$ & $35(57.4)$ & 0.650 \\
\hline Diuretics n (\%) & $169(74.1)$ & $64(37.9)$ & $105(62.1)$ & 0.021 \\
\hline Vasodilators n (\%) & $26(I 1.4)$ & $9(34.6)$ & $17(65.4)$ & 0.409 \\
\hline Digoxin n (\%) & $95(41.7)$ & $58(6 I .1)$ & $37(38.9)$ & 0.002 \\
\hline Anticoagulants n (\%) & $7 I(3 I .1)$ & $28(39.4)$ & $43(60.6)$ & 0.565 \\
\hline Antiplatelet n (\%) & $69(30.3)$ & $23(33.3)$ & $46(66.7)$ & 0.080 \\
\hline Inotropic agents n (\%) & $19(8.3)$ & $7(26.8)$ & $12(63.2)$ & 0.638 \\
\hline Statins n (\%) & $73(32.0)$ & $30(4 I .1)$ & $43(58.9)$ & 0.776 \\
\hline \multicolumn{5}{|l|}{ Medication during discharged } \\
\hline ACE inhibitors $\mathrm{n}(\%)$ & $97(42.5)$ & $40(4 I .2)$ & $57(58.8)$ & 0.787 \\
\hline ARBs n (\%) & $52(22.8)$ & $27(51.9)$ & $25(48.1)$ & 0.151 \\
\hline Beta-blockers n (\%) & $102(44.7)$ & $4 I(40.2)$ & $6 \mathrm{I}(59.8)$ & 0.030 \\
\hline Diuretics n (\%) & $147(64.5)$ & $54(36.7)$ & $93(63.3)$ & 0.018 \\
\hline Vasodilators n (\%) & $10(4.4)$ & $6(60.0)$ & $(40.0)$ & 0.747 \\
\hline Digoxin n (\%) & $56(24.7)$ & $22(39.3)$ & $34(60.7)$ & 0.641 \\
\hline Anticoagulants n (\%) & $77(33.8)$ & $32(42.1)$ & $44(58.9)$ & 0.925 \\
\hline Antiplatelet n (\%) & $95(4 I .7)$ & $36(37.9)$ & $59(62.1)$ & 0.277 \\
\hline Statins n (\%) & $80(35.1)$ & $30(4 I .1)$ & $43(58.9)$ & 0.779 \\
\hline
\end{tabular}

Abbreviations: HF, heart failure; ADCHF, acute decompensated chronic heart failure; ACEls, angiotensin-converting enzyme inhibitors; ARBs, angiotensin receptor Blockers.

Table 4 Binary and Multivariate Logistic Regression Analysis to Identify Factors Associated Within Long-Term Hospital Stay Among Heart Failure Patients at DMCSH

\begin{tabular}{|c|c|c|c|c|}
\hline Variables & COR $(95 \% \mathrm{Cl})$ & P-value & AOR $(95 \% \mathrm{Cl})$ & P-value \\
\hline Systolic blood pressure $(\mathrm{mmHg})$ & $1.11(1.00-1.23)$ & 0.057 & $1.26(0.99-1.53)$ & 0.006 \\
\hline Serum creatinine $(\mathrm{mg} / \mathrm{dl}$ & $0.5 I(0.34-0.76)$ & 0.001 & $0.46(0.27-0.87)$ & 0.004 \\
\hline \multicolumn{5}{|l|}{ Heart failure category } \\
\hline $\mathrm{ADCHF}$ & $2.03(1.18-3.48)$ & 0.010 & $2.66(1.35-5.22)$ & 0.005 \\
\hline New onset (de novo) HF & 1.00 & 1.00 & 1.00 & 1.00 \\
\hline \multicolumn{5}{|l|}{ Diabetes mellitus } \\
\hline Yes & $2.40(1.13-5.11)$ & 0.023 & $2.07(0.99-4.33)$ & 0.025 \\
\hline No & 1.00 & 1.00 & 1.00 & 1.00 \\
\hline \multicolumn{5}{|l|}{ Class of medication ARBs } \\
\hline Yes & $1.69(0.95-3.00)$ & 0.074 & $2.32(1.03-5.20)$ & 0.042 \\
\hline No & 1.00 & 1.00 & 1.00 & 1.00 \\
\hline
\end{tabular}

Abbreviations: HF, heart failure; ADCHF, acute decompensated chronic heart failure; DMCSH, Debre Markos Comprehensive Specialized Hospital; ARBs, angiotensin receptor blockers; COR, crude odd ratio; AOR, adjusted odd ratio.

reduction in ejection fraction and higher prevalence of atrial fibrillation among de novo heart failure patients than ADCHF in this study. In addition, this finding was consistent with the recommendation of the 2017 ACCF/
AHA guideline for the management of HF patients with reduced ejection fraction. ${ }^{25}$ Beta-blockers were the second most prescribed discharge medications following diuretics, which were also significantly higher among the ADCHF 
group. This was in line with other similar studies. ${ }^{13,22}$ This might be because beta-blockers are among groups of medications that have survival benefits and should be added after patients are thermodynamically stabilized.

The median hospital stay found in this study was 12 days (IQR 8-18). This finding was closely similar to studies conducted in Portugal (11 days), Italy (10 days), and a previous study at St. Paul's Hospital Millennium Medical College in Addis Ababa, Ethiopia (11 days). ${ }^{8,12,16}$ However, it was higher than studies reported in Gulf countries (6 days), India (6 days) California, and Los Angeles (4 days). ${ }^{13,24,25}$ The finding of the present study was also higher than studies conducted in Western Romania (8 days) and Tikur Anbessa Specialized Hospital, Ethiopia (4 days). ${ }^{17,26}$ This difference might be due to the small used sample size at Western Romania and Tikur Anbessa specialized hospital, Ethiopia (71 vs 169, respectively). In addition, at Tikur Anbessa Specialized Hospital study participants were younger (mean $\pm \mathrm{SD}$, $37.5 \pm 17.8$ as compared to $53.31 \pm 15.68$ in this study).

HF patients at risk for longer lengths of stay (LOS) are a very important target of intervention for care providers to achieve a better outcome. Increasing systolic blood pressure was significantly associated with longer LOS as compared to patients within normal systolic blood pressure. This might be because uncontrolled hypertension is the main cause of left ventricular hypertrophy, where the heart muscle is weakened, which affects its ability to pump properly which may in turn acutely exacerbate HF and contribute to hospitalization for a long time. ${ }^{11,16}$ Patients with $\mathrm{ADCHF}$ and the presence of DM comorbidity were also significantly associated with longer LOS. It has been recognized both cardiovascular and non-cardiovascular comorbidities including DM are predictors of hospitalizations, mortality, and longer hospital stay. ${ }^{17,24}$ On the other hand, lower serum creatinine concentration was significantly associated with shorter LOS as compared to elevated serum creatinine. ${ }^{27}$ In another way, a study conducted at the Cardiology Unit of Altamura Hospital, Italy showed that the presence of signs of congestion, including blood urea nitrogen to creatinine $(\mathrm{BUN} / \mathrm{Cr}$ ) values above the calculated cut-off was linked to an adverse prognosis in HF patients. ${ }^{28}$

In the present study, the use of ARBs was significantly increased LOS. Previous randomized controlled trials (RCTs) established that ARBs reduce morbidity, promote regression of ventricular hypertrophy, and reduce the incidence of sudden cardiac death and indeed the length of hospital stay. ${ }^{13,27}$ However, in this study, the reverse is true possibly due to the cost and difficulty of accessibility of ARBs, leading to poor treatment compliance. In addition, the use of ARBs might be resulting in worsening kidney function in both acute de novo HF and ADCHF patients. ${ }^{25}$ Therefore, assessment of kidney function including glomerular filtration rate becomes mandatory in the management of patients with $\mathrm{HF}^{29}$ This might be required additional time for further investigation and increase length of hospital stay among HF patients. This observation highlights the importance of developing and implementing quality improvement programs in the evidence-based management of HF.

\section{Limitations of the Study}

The study was conducted in one setting where; the findings might not help to reflect the health-care conditions of other health settings in Ethiopia. Further, we did not systematically capture data on drug doses during hospitalization or at the time of discharge and follow-up, which might have been useful for creating better risk prediction models. Another limitation of the study was the failure of considering appropriateness and adherence to previous drug therapy among ADCHF. In addition, quality of life including daily activity measurement and nutritional status might be affected readmission and length of hospital stay in HF patients. ${ }^{30}$ However, this study is unable to address these factors. Despite these limitations, the present prospective cohort study provides new insights into admission variables difference between de novo and ADCHF as well as predictive of LOS.

\section{Conclusion}

Acute heart failure is a major public health problem presented with diverse clinical characteristics. Length of hospital stay is still high among hospitalized heart failure patients, and duration of hospitalization was higher in patients with ADCHF. Moreover, patients with ADCHF, increasing SBP, the use of ARBs, and the presence of DM co-morbidity were independent predictors of long-term hospital stay. On the other hand, low mean serum creatinine concentration during the index of admission was associated with a short duration of hospital stay. Diuretics were the most commonly prescribed medications during admission and at the time of discharge. During hospitalization, digoxin and ARBs were the most frequently prescribed class of medication in de novo $\mathrm{HF}$ while beta-blockers were significantly higher in ADCHF 
at discharge. Initiation of treatment in clinical practice must take into account the heterogeneity of each patient, which ultimately may help improve patient outcomes. In addition, more researches or large surveys addressing more heart failure patients in a different clinical setting requires assessing the overall situation.

\section{Abbreviations}

ACEIs, angiotensin-converting enzyme inhibitors; ADCHF, acute decompensated chronic heart failure; ARBs, angiotensin receptor blocker; CAD, coronary artery disease; CI, confidence interval; COPD, chronic obstructive pulmonary disease; CVD, cardiovascular disorder; DMCSH, Debre Markos Comprehensive specialized Hospital; LOS, length of hospital stay; NCD, noncommunicable diseases; NYHA, New York Health Association.

\section{Data Sharing Statement}

The data sets used and/or analyzed during the current study are available from the corresponding author on reasonable request.

\section{Ethics Approval and Consent to Participate}

The Ethical Reviewer committee, College of Health Science Debre Markos University (HSC/R/C/Ser/Co/347/ 06/12), approved this study. This study was conducted in accordance with the Declaration of Helsinki in which it is stated that in medical research using identifiable human material or data, physicians must normally seek consent for the collection, analysis, storage and/or reuse. Before data collection, individual written consent was obtained from the study participants or caregivers. Both of them were informed about the objective of the study, procedures of selection, and assurance of confidentiality, and their names were not registered to minimize social desirability bias and enhance anonymity. Patients were not forced to participate and received any monetary incentive and it was solely voluntary based. The collected data was handled and secured with the principal investigator on every data collection day.

\section{Acknowledgments}

We thank the participants of the study and Debre Markos University.

\section{Author Contributions}

All authors made substantial contributions to conception and design, acquisition of data, or analysis and interpretation of data; took part in drafting the article or revising it critically for important intellectual content; agreed to submit to the current journal; gave final approval of the version to be published; and agreed to be accountable for all aspects of the work.

\section{Funding}

This research was funded by Debre Markos University, College of Health Sciences. The University has no role in designing, conducting, and reporting the study.

\section{Disclosure}

The authors declared that they have no conflicts of interest in this work.

\section{References}

1. Bloomfield GS, Barasa FA, Doll JA, Velazquez EJ. Heart failure in sub-Saharan Africa. Curr Cardiol Rev. 2013;9(2):157-173. doi:10.2174/1573403X11309020008

2. Savarese G, Lund LH. The global public health burden of heart failure. Card Fail Rev. 2017;3(1):7. doi:10.15420/cfr.2016:25:2

3. Dokainish H, Teo K, Zhu J, et al. Heart failure in Africa, Asia, the Middle East, and South America: the INTER-CHF study. Int J Cardiol. 2016;204:133-141. doi:10.1016/j.ijcard.2015.11.183

4. Shiferaw F, Letebo M, Misganaw A, et al. Non-communicable Diseases in Ethiopia: disease burden, gaps in health care delivery and strategic directions. Ethiop J Health Dev. 2018;32(3).

5. Tefera YG, Abegaz TM, Abebe TB, Mekuria AB. The changing trend of cardiovascular disease and its clinical characteristics in Ethiopia: a hospital-based observational study. Vasc Health Risk Manag. 2017;13:143. doi:10.2147/VHRM.S131259

6. Abdissa SG, Oli K, Feleke Y, Goshu DY, Begna DM, Tafese A. Spectrum of cardiovascular diseases among Ethiopia patients at Tikur anbessa specialized university teaching hospital, Addis Ababa. Ethiop Med J. 2014;52(1):9-17.

7. Habte B, Alemseged F, Tesfaye D. The pattern of cardiac diseases at the cardiac clinic of Jimma University specialized hospital, South West Ethiopia. Ethiop J Health Sci. 2010;20(2):99-105.

8. Diaz A, Ciocchini C, Esperatti M, Becerra A, Mainardi S, Farah A. Precipitating factors leading to decompensation of chronic heart failure in the elderly patient in South-American community hospital. J Geriatr Cardiol. 2011;8(1):12. doi:10.3724/SP.J.1263.2011.00012

9. Pinho-Gomes AC, Cardoso JS, Azevedo LF, Almeida R, Pinho T, Maciel MJ. Characterization of acute heart failure hospitalizations in a Portuguese cardiology department. Revista Portuguesa de Cardiologia. 2013;32(7-8):567-575. doi:10.1016/j.repc.2012.10.018

10. Crişan S, Petrescu L, Lazăr MA, et al. Reduced ejection fraction heart failure-new data from multicenter studies and national registries regarding general and elderly populations: hopes and disappointments. Clin Interv Aging. 2018;13:651. doi:10.2147/CIA.S161385

11. Follath F, Yilmaz M, Delgado J, et al. Clinical presentation, management and outcomes in the acute heart failure global survey of standard treatment (ALARM-HF). Intensive Care Med. 2011;37 (4):619-626. doi:10.1007/s00134-010-2113-0 
12. Oliva F, Mortara A, Cacciatore G, et al. Acute heart failure patient profiles, management and in-hospital outcome: results of the Italian Registry on Heart Failure Outcome. Eur J Heart Fail. 2012;14 (11):1208-1217. doi:10.1093/eurjhf/hfs117

13. Sulaiman K, Panduranga P, Al-Zakwani I, et al. Clinical characteristics, management, and outcomes of acute heart failure patients: observations from the Gulf acute heart failure registry (Gulf CARE). Eur J Heart Fail. 2015;17(4):374-384. doi:10.1002/ejhf.245

14. Franco J, Formiga F, Corbella X, et al. De novo acute heart failure: clinical features and one-year mortality in the Spanish nationwide Registry of Acute Heart Failure. Medicina Clínica. 2019;152 (4):127-134. doi:10.1016/j.medcle.2018.12.009

15. Lassus JP, Siirilä-Waris K, Nieminen MS, et al. Long-term survival after hospitalization for acute heart failure-differences in prognosis of acutely decompensated chronic and new-onset acute heart failure. Int J Cardiol. 2013;168(1):458-462. doi:10.1016/j.ijcard.2012.09.128

16. Woldeyes Asfaw E. Five years Clinical characteristics and in-hospital outcome of acute heart failure at tertiary care hospital in Ethiopia. Ethiop Med J. 2020;58(01).

17. Tirfe M, Nedi T, Mekonnen D, Berha AB. Treatment outcome and its predictors among patients of acute heart failure at a tertiary care hospital in Ethiopia: a prospective observational study. BMC Cardiovasc Disord. 2020;20(1):1-10. doi:10.1186/s12872-019-01318-x

18. Mwita JC, Dewhurst MJ, Magafu MG, et al. Presentation and mortality of patients hospitalized with acute heart failure in Botswana. Cardiovasc J Afr. 2017;28(2):112-117. doi:10.5830/CVJA-2016-067

19. Loop MS, Van Dyke MK, Chen L, et al. Comparison of length of stay, 30-day mortality, and 30-day readmission rates in Medicare patients with heart failure and with reduced versus preserved ejection fraction. Am J Cardiol. 2016;118(1):79-85. doi:10.1016/j.amjcard.2016.04.015

20. Román-Sánchez P, Conthe P, García-Alegría J, Forteza-Rey J, Montero M, Montoto C. Factors influencing the medical treatment of heart failure patients in Spanish internal medicine departments: a national survey. Qjm. 2005;98(2):127-138. doi:10.1093/qjmed/hci019

21. Kapoor JR, Kapoor R, Ju C, et al. Precipitating clinical factors, heart failure characterization, and outcomes in patients hospitalized with heart failure with reduced, borderline, and preserved ejection fraction. JACC Heart Fail. 2016;4(6):464-472.
22. AlHabib KF, Kashour T, Elasfar AA, et al. Long-term mortality rates in acute de novo versus acute-on-chronic heart failure: from the heart function assessment registry trial in Saudi Arabia. Angiology. 2015;66(9):837-844. doi:10.1177/0003319714563138

23. Harikrishnan S, Sanjay G, Anees T, et al. Clinical presentation, management, in-hospital and 90-day outcomes of heart failure patients in Trivandrum, Kerala, India: the Trivandrum Heart Failure Registry. Eur J Heart Fail. 2015;17(8):794-800. doi:10.1002/ejhf.283

24. Whellan DJ, Zhao X, Hernandez AF, et al. Predictors of hospital length of stay in heart failure: findings from getting With the Guidelines. J Card Fail. 2011;17(8):649-656. doi:10.1016/j.cardfail.2011.04.005

25. Filippatos GS MH, Fonarow G, Givertz M, et al. 2017 ACC/AHA/ HFSA focused update of the 2013 ACCF/AHA guideline for the management of heart failure. Circulation. 2017;136:e137-e161.

26. Gyalai-Korpos I, Ancusa O, Dragomir T, Tomescu MC, Marincu I. Factors associated with prolonged hospitalization, readmission, and death in elderly heart failure patients in western Romania. Clin Interv Aging. 2015;10:561.

27. Panduranga P, Sulaiman K, Al-Zakwani I, et al. Demographics, clinical characteristics, management, and outcomes of acute heart failure patients: observations from the Oman acute heart failure registry. Oman Med J. 2016;31(3):188. doi:10.5001/omj.2016.37

28. Massari F, Scicchitano P, Iacoviello M, et al. Multiparametric approach to congestion for predicting long-term survival in heart failure. J Cardiol. 2020;75(1):47-52. doi:10.1016/j.jjcc.2019.05.017

29. Scicchitano P, Iacoviello M, Passantino A, et al. The prognostic impact of estimated creatinine clearance by bioelectrical impedance analysis in heart failure: comparison of different eGFR formulas. Biomedicines. 2021;9(10):1307. doi:10.3390/biomedicines9101307

30. Kitamura M, Izawa KP, Yaekura M, et al. Relationship among activities of daily living, nutritional status, and 90-day readmission in elderly patients with heart failure. Int J Environ Res Public Health. 2019;16(24):5068. doi:10.3390/ijerph16245068
Research Reports in Clinical Cardiology

\section{Publish your work in this journal}

Research Reports in Clinical Cardiology is an international, peerreviewed, open access journal publishing original research, reports, editorials, reviews and commentaries on all areas of cardiology in the clinic and laboratory. The manuscript management system is

\section{Dovepress}

completely online and includes a very quick and fair peer-review system. Visit http://www.dovepress.com/testimonials.php to read real quotes from published authors. 